\title{
The Diuretic Effect of Sacubitril/Valsartan Might Be Clinically Relevant
}

\author{
Luís Beck-da-Silvat,2 and Luís E. Rohde ${ }^{1,2}$ \\ Serviço de Cardiologia - Hospital de Clínicas de Porto Alegre, ${ }^{1}$ Porto Alegre, RS - Brazil \\ Programa de Pós-Graduação em Cardiologia e Ciências Cardiovasculares - Faculdade de Medicina da Universidade Federal do Rio Grande do \\ Sul, ${ }^{2}$ Porto Alegre, $R S-$ Brazil
}

Recently, patients with heart failure have been prescribed a novel and innovative drug. Sacubitril/valsartan is a new drug modality that brings a $16 \%$ reduction in total mortality, a $20 \%$ reduction in cardiovascular mortality and $21 \%$ reduction in hospital admissions due to heart failure. The benefit is undoubtedly clinically relevant and the clinical trial which have shown such benefit have achieved an unprecedented statistical significance. ${ }^{1}$

The mechanism of action of sacubitril/valsartan combines the well-known vasodilatory effect of valsartan associated with the neutral endopeptidase (NEP) inhibition effect of sacubitril, which will ultimately result in increased serum levels of natriuretic peptides, increased action of endogenous natriuretic peptides in target tissues by prolonging its tissue half-life, and consequently increased vasodilatory, anti-proliferative and natriuretic effects. ${ }^{1}$

Although the current approach of replacing enalapril with sacubitril/valsartan might sound as a switch of vasodilators in patients with heart failure, the addition of natriuretic effect provided by sacubitril may in fact be the driving force of the clinical benefits. In favor of this concept we can make a few comments:

a. Hypotension, more frequently seen in sacubitril/ valsartan than in the enalapril group, could possibly be associated with hypovolemia caused by the natriuretic effect of sacubitril;

b. Patients who received valsartan (160 mg twice daily) in the Val-HEFT trial ${ }^{2}$ did not show the same benefit on mortality or on hypotensive adverse events as those demonstrated in the PARADIGM-HF trial (sacubitril/ valsartan 97/103 mg twice daily).

c. A post hoc analysis of data from the PARADIGM-HF study revealed that the increase in the mean dose of furosemide was smaller in the sacubitril/valsartan group

\section{Keywords}

Heart Failure; Valsartan/therapeutic use; Aminobutyrates/ pharmacology; Angiotensin Receptor Antagonists/pharmacology; Natriuretic Peptides/physiology; Tetrazoles/farmacologia.

Mailing Address: Luís Beck-da-Silva

Hospital de Clínicas de Porto Alegre - Rua Ramiro Barcelos, 2350, Sala 2060. CEP 90035-903, Porto Alegre, RS - Brazil

E-mail: Ibneto@hcpa.edu.br, luisbeckdasilva@gmail.com

Manuscript received August 27, 2018 revised manuscript September 30, 2018, accepted October 24, 2018 compared with the enalapril group, and that the median dose of furosemide increased in the enalapril group, but not in the sacubitril/valsartan group. ${ }^{3}$

It is well known from observational studies and metaanalyses that increased doses of diuretics have been linked to worse prognosis in patients with heart failure. Despite inherent biases associated with observational studies, it is biologically plausible that diuretics are potentially harmful due to heir hyperreninemic, vasoconstrictive and hypokalemic effects. One of the few clinical trials conducted on diuretics in patients with heart failure, the DOSE trial, have shown greater kidney toxicity associated with higher doses of furosemide. Diuretic dose reduction associated with sacubitril/valsartan therapy might be a desired secondary effect of this compound in patients with heart failure. ${ }^{4,5}$

In that sense, studies on diuretic withdrawal are mostly needed. The REBIC (REde Brasileira de Insuficiênia Cardíaca - Heart Failure Brazilian Network) trial is under way and is intended to be the largest clinical trial ever conducted designed to assess the effects of diuretic withdrawal in ambulatory patients with heart failure. ${ }^{6}$ A subgroup of patients on sacubitril-valsartan will be compared with those on angiotensin-converting enzyme inhibitors/angiotensin receptor blocker for tolerance of diuretic withdrawal.

While no other data are available, it is reasonable to recommend closer attention to patients' volume status and exercise a low threshold to decrease or even discontinue diuretics in heart failure patients on sacubitril/valsartan.

\section{Author contributions}

Conception and design of the research and Writing of the manuscript:: Beck-da-Silva L; Critical revision of the manuscript for intellectual content: Beck-da-Silva L, Rohde LE.

\section{Potential Conflict of Interest}

No potential conflict of interest relevant to this article was reported.

\section{Sources of Funding}

There were no external funding sources for this study.

\section{Study Association}

This study is not associated with any thesis or dissertation work.

DOI: $10.5935 / a b c .20190080$ 


\section{References}

1. McMurray JJ, Packer M, Desai AS, Gong J, Lefkowitz MP, Rizkala AR, et al. Angiotensin-neprilysin inhibition versus enalapril in heart failure. N Engl J Med. 2014;371(11):993-1004.

2. Cohn JN, Tognoni G, the Valsartan Heart Failure Trial I. A randomized trial of the angiotensin-receptor blocker Valsartan in chronic heart failure. N Engl J Med. 2001;345(23):1667-75.

3. Ayalasomayajula S, Schuehly U, Pal P, Chen F, Zhou W, Sunkara G, et al. Effect of the angiotensin receptor-neprilysin inhibitor sacubitril/valsartan on the pharmacokinetics and pharmacodynamics of a single dose of furosemide. Br J Clin Pharmacol. 2018;84(5):926-36.
4. Yilmaz MB, Gayat E, Salem R, Lassus J, Nikolaou M, Laribi M, et al. Impact of diuretic dosing on mortality in acute heart failure using a propensity-matched analysis. Eur J Heart Fail. 2011;13(11):1244-52.

5. Felker GM, Lee KL, Bull DA, Redflield MM, Stevenson LW, Goldsmith SR, et al. et al. Diuretic Strategies in Patients with Acute Decompensated Heart Failure. N Engl L Med. 2011;364(9):797-805.

6. da Rosa PR, Rohde LE, Doebber M, Ribeiro ALP, Prado DP, Bertoldi FG, et al Rational and design of a randomized, double-blind, multicenter trial to evaluate the safety and tolerability of furosemide withdrawal in stable chronic outpatients with heart failure: The ReBIC-1 trial. Am Heart J. 2017 Dec ;194:125-31. 